\title{
L'entrepreneuriat technologique : ses parties prenantes et leurs implications
}

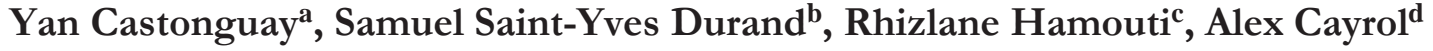

RÉSUMÉ. Étant moins bien connu et documenté que l'entrepreneuriat conventionnel, l'entrepreneuriat technologique se distingue par son processus d'innovation gravitant principalement autour de la création d'une technologie. Ce projet de recherche vise à mieux comprendre le rôle des parties prenantes au sein de l'écosystème entrepreneurial lors de projets d'innovation technologique. Basées sur une approche exploratoire, 11 entrevues semi-structurées ont été réalisées en 2017 auprès d'entrepreneurs qui créent des technologies diverses dans la province de Québec au Canada. L'analyse de ces entrevues a permis d'identifier les implications des parties prenantes dans les trois phases du processus d'innovation technologique : la conception, la mise en œuvre et le marketing. Par conséquent, cette recherche fournit non seulement des recommandations pour les entrepreneurs technologiques, mais aussi des pistes de recherche.

ABSTRACT. Being less known and documented than conventional entrepreneurship, technological entrepreneurship can be distinguished by its innovation process that revolves around the creation of a technology. This research project is intended to belp to better understand the role of the stakeholders within the entrepreneurial ecosystem in technological innovation projects. Based on an exploratory approach, 11 semi-structured interviens were conducted in 2017 with entrepreneurs who create various technologies in the province of Quebec, Canada. The analysis of these interviews allowed to identify the participation of the stakeholders in the three phases of the technological innovation process: conception, implementation and marketing. Consequently, this research provides not only recommendations for technological entrepreneurs, but also clues for research.

\section{Introduction}

L'entrepreneuriat technologique joue un rôle crucial au cœur du développement économique du xxie siècle, notamment en ce qui a trait à la création d'industries et même à la sauvegarde d'industries en déclin. Depuis plusieurs années, le champ de recherche portant sur l'entrepreneuriat s'intéresse à la découverte et à l'exploitation d'opportunités profitables (Shane et Venkataraman, 2003). Le savoir-faire et la créativité d'individus qu'on qualifie d'entrepreneurs permettent d'exploiter ces opportunités.

Parmi ces opportunités, certaines nécessitent la maitrise de technologies de pointe. Or, de plus en plus d'entrepreneurs développent des innovations technologiques. Ainsi, l'entrepreneuriat technologique représente une nouvelle tendance au sein de l'écosystème entrepreneurial. Étant unique et singulier, il se distingue de l'entrepreneuriat traditionnel, non seulement par l'importance accordée aux avancées des connaissances technologiques et scientifiques qui seront réalisées, mais également par son aspect moins linéaire et prévisible. En effet, ce type d'entrepreneuriat est souvent peu planifié et émerge de façon imprévisible. Il est souvent le fruit d'échanges entre plusieurs parties prenantes.

À ce sujet, l’analyse de la littérature a révélé que peu d'auteurs ont étudié l'implication de diverses parties prenantes dans le processus d'innovation technologique. En ce sens, et en raison de son importance pour le développement économique actuel et étant

\footnotetext{
${ }^{a}$ Professeur, Ph. D., Université du Québec à Rimouski

${ }^{\mathrm{b}}$ Professeur, M.B.A., CPA auditeur, CA, Université du Québec à Rimouski

${ }^{\text {C }}$ Professeur, Ph. D., Université du Québec en Outaouais

d Candidat au doctorat, Grenoble École de management
} 
donné que ce concept est relativement nouveau, l'entrepreneuriat technologique nécessite une meilleure compréhension, particulièrement en ce qui a trait à son processus d'innovation.

Cet article définit dans un premier temps le concept de l'entrepreneuriat technologique, incluant l'écosystème entrepreneurial, les parties prenantes et son processus d'innovation. Les questions de recherche ainsi que la méthodologie sont par la suite présentées. Les résultats des entrevues sont analysés en fonction des trois phases du processus d'innovation. Finalement, l'article terminera avec une discussion des résultats visant à susciter une réflexion auprès des praticiens et chercheurs.

\section{Entrepreneuriat technologique}

Dans une revue de littérature portant sur l'entrepreneuriat technologique, Bailetti (2012) a recensé 93 articles scientifiques publiés entre 1970 et 2011 qui ont abordé ce sujet. Il a ainsi identifié les six définitions de ce concept. La première définition est celle de Jones-Evans (1995), qui définit l'entrepreneuriat technologique comme une création d'une nouvelle entreprise technologique. Jelinek (1996), quant à elle, souligne l'importance d'une compréhension commune et des efforts de coordination nécessaires afin de réaliser ces changements technologiques. En 2001, Venkataraman et Sarasvathy ont traité des défis de ce type d'entrepreneuriat en précisant que l'entrepreneuriat technologique cherche des solutions à des problèmes souvent liés à la technologie.

Par la suite, la notion de risque a été abordée dans la littérature. En effet, Nichols et Armstrong (2003) définissent l'entrepreneuriat technologique comme une organisation, une gestion et une prise de risque axées sur la technologie. Garud et Karnøe (2003) ajoutent à cette définition l'implication de parties prenantes visant à collaborer au sein d'un projet d'entrepreneuriat technologique. Finalement, la définition de Liu, Chu, Hung et Wu (2005) traite des moyens par lesquels les entrepreneurs utilisent les ressources et les structures pour exploiter les opportunités technologiques émergentes.

En se basant sur l'analyse de ces six définitions, Bailetti (2012) définit l'entrepreneuriat technologique comme étant «un investissement dans un projet qui rassemble et déploie des individus spécialisés et des actifs hétérogènes étroitement liés aux avancées des connaissances scientifiques et technologiques dans le but de créer et de générer de la valeur pour une entreprise » (p. 5, trad. libre).

\section{1 Écosystème entrepreneurial}

L'entrepreneuriat technologique s'exerce à l'intérieur d'un écosystème entrepreneurial dont les parties prenantes viennent influencer les projets. Bien qu'il existe de nombreuses définitions du concept d'écosystème entrepreneurial (Muldoon, Bauman et Lucy, 2018), la plupart des auteurs mettent l'accent sur l'implication et les interactions formelles ou informelles des parties prenantes dans le processus d'innovation. Selon Mason et Brown (2014), l'écosystème entrepreneurial fournit un milieu propice aux échanges entre ces différents acteurs.

\subsection{Parties prenantes}

Dans son Guide PMBOK, le Project Management Institute (2008) définit les parties prenantes comme étant des « personnes et organisations, telles que les clients, les commanditaires, les entreprises réalisatrices du projet et le public, activement impliquées dans le projet ou dont les intérêts peuvent être affectés de manière positive ou négative par l'exécution ou l'achèvement du projet. Les parties prenantes peuvent également influencer le projet et ses livrables » (p. 438, trad. libre). Chacune de ces parties prenantes peut donc jouer un rôle différent dans le processus d'innovation technologique.

\subsection{Processus d'innovation}

L'entrepreneuriat technologique repose essentiellement sur un projet d'innovation (Bailetti, 2012; Shane et Venkataraman, 2003). Par conséquent, il est pertinent d'identifier les différentes phases de ce type de projet. Pour ce faire, le choix d'un cadre d'analyse permettant de comprendre les différents rôles des parties prenantes est essentiel. Bien que le processus d'innovation ne soit pas toujours linéaire, la revue systématique de littérature effectuée par Wirtz et Daiser (2018) basée sur 20 approches distinctives entre 2000 et 2014 a permis d'identifier un modèle générique composé de 7 phases dans le processus d'innovation des modèles d'affaires: l'analyse, l'idéation, la faisabilité, le prototypage, la prise de décision, la mise en œuvre et la durabilité. Ce modèle générique de Wirtz et Daiser (2018) fournit de nombreuses informations sur les activités de chacune de ces phases. Bien que la mise en 
marché et le lancement d'un nouveau projet représentent des activités importantes pour la commercialisation d'un projet d'innovation, le modèle de Wirtz et Daiser (2018) n'aborde pas ou peu cette phase de marketing.

Le processus simplifié d'innovation de Tiwari (2007) demeure, quant à lui, plus concis que celui de Wirtz et Daiser (2018). Il concentre les activités du processus d'innovation en trois phases : la conception, la mise en œuvre et le marketing.
Le tableau 1 présente les phases des modèles de Wirtz et Daiser (2018) et de Tiwari (2007) ainsi que les activités de chacune de ces phases. Il est à noter que la phase de durabilité du modèle de Wirtz et Daiser touche à la fois aux phases de mise en œuvre et de marketing du modèle de Tiwari. La simplicité du modèle de Tiwari regroupant les activités d'un projet d'innovation en trois phases nous permettra d'étudier l'entrepreneuriat technologique de manière à mieux répondre aux questions de recherche.

\begin{tabular}{|c|c|}
\hline $\begin{array}{l}\text { Processus d'innovation des modèles d'affaires } \\
\text { (Wirtz et Daiser, 2018) } \\
\text { Phases et activités }\end{array}$ & $\begin{array}{l}\text { Processus simplifié } \\
\text { d'innovation } \\
\text { (Tiwari, 2007) } \\
\text { Phases et activités }\end{array}$ \\
\hline $\begin{array}{l}\text { 1. Analyse } \\
\text { - Analyse des modèles d'affaires actuels } \\
\text { - Analyse des produits et services } \\
\text { - Analyse des clients et groupes ciblés } \\
\text { - Analyse des marchés et de la compétition }\end{array}$ & \multirow{3}{*}{$\begin{array}{l}\text { 1. Conception } \\
\text { - Analyse des besoins } \\
\text { - Génération d'idées } \\
\text { - Évaluation d'idées } \\
\text { - Planification du projet }\end{array}$} \\
\hline $\begin{array}{l}\text { 2. Idéation } \\
\text { - Détermination de la mission des modèles d'affaires d'innovation } \\
\text { - Génération de connaissances sur les clients } \\
\text { - Développement de scénarios de clients } \\
\text { - Pensée visuelle/réseau et narration }\end{array}$ & \\
\hline $\begin{array}{l}\text { 3. Faisabilité } \\
\text { - Hypothèses sur l'environnement des affaires } \\
\text { - Analyse des interdépendances } \\
\text { - Analyse de l'alignement potentiel du modèle d'entreprise interne ou externe }\end{array}$ & \\
\hline $\begin{array}{l}\text { 4. Prototypage } \\
\text { - Analyse de différents designs de modèles d'affaires d'innovation alternatifs } \\
\text { - Création de différents designs de modèles d'affaires d'innovation alternatifs } \\
\text { - Développement de plusieurs concepts détaillés } \\
\text { - Raffinement des composants/modèles partiels }\end{array}$ & \multirow{4}{*}{$\begin{array}{l}\text { 2. Mise en œuvre } \\
\text { - Développement/ } \\
\text { construction } \\
\text { - Prototype } \\
\text { - Application d'un pilote } \\
\text { - Test }\end{array}$} \\
\hline $\begin{array}{l}\text { 5. Prise de décision } \\
\text { - Évaluation de chaque design de modèle d'affaires d'innovation alternatif } \\
\text { - Sélection du design final de modèle d'affaires d'innovation } \\
\text { - Harmonisation finale des composants } \\
\text { - Réalisation et test du modèle d'affaires d'innovation }\end{array}$ & \\
\hline $\begin{array}{l}\text { 6. Mise en œuvre } \\
\text { - Développement du plan de la mise en œuvre } \\
\text { - Communication et construction de l'équipe } \\
\text { - Réalisation du modèle d'affaires d'innovation étape par étape } \\
\text { - Achèvement de la mise en œuvre }\end{array}$ & \\
\hline 7. Durabilité & \\
\hline $\begin{array}{l}\text { - Surveillance et contrôle du modèle d'affaires d'innovation } \\
\text { - Adaptation potentielle du modèle d'affaires d'innovation } \\
\text { - Croissance soutenue grâce à l'apprentissage à l'échelle de l'organisation } \\
\text { - Création de mécanismes d'isolement face à la concurrence } \\
\text { - Obtention d'un avantage concurrentiel à long terme } \\
\text { - Transition du modèle d'affaires d'innovation }\end{array}$ & $\begin{array}{l}\text { 3. Marketing } \\
\text { - Production } \\
\text { - Pénétration de marché } \\
\text { - Lancement (national et in- } \\
\text { ternational) }\end{array}$ \\
\hline
\end{tabular}

Tableau 1 - Processus d'innovation

Source : Wirtz et Daiser, 2018 et Tiwari, 2007 (trad. libre) 


\section{Objectifs de recherche}

L'objectif général de cette recherche est de mieux comprendre l'entrepreneuriat technologique et, plus spécifiquement, le rôle des parties prenantes dans le processus d'innovation technologique. Pour ce faire, il sera important de répondre à deux questions spécifiques :

1. Quelles sont les parties prenantes impliquées dans chacune des phases du processus d'innovation (conception, mise en œuvre et marketing)?

2. Quelles sont les implications des parties prenantes dans chacune de ces phases du processus d'innovation?

\section{Méthodologie}

Afin de répondre aux objectifs de cette recherche, une approche exploratoire est adoptée (Miles et Huberman, 2005; Thiétart, 2014). Par sa démarche inductive, cette approche permet de mieux saisir le phénomène étudié, soit l'entrepreneuriat technologique (Gauthier et Bourgeois, 2016). Dans un premier temps, la méthodologie de ce projet de recherche a été validée par le Comité d'éthique de la recherche de l'Université du Québec à Rimouski. Ensuite, un échantillonnage de convenance auprès d'entrepreneurs technologiques dans la province de Québec a été identifié avec l'aide d'organismes gouvernementaux de soutien.

Au final, 11 entrevues semi-structurées ont été conduites auprès des entrepreneurs entre janvier et avril 2017. Parmi ces entrepreneurs, 1 entreprise se situait dans la phase de conception du processus simplifié d'innovation, 4 entreprises se situaient dans la phase de mise en œuvre et 6 entreprises avaient atteint la phase de marketing. D'une durée moyenne de 1 heure, chacune des entrevues a été enregistrée sur un support numérique et a été retranscrite par la suite. Les verbatim de ces entrevues ont été codifiés en utilisant le logiciel NVivo version 11. L'analyse des données a été effectuée par quatre chercheurs de manière à réaliser une analyse thématique permettant de répondre aux objectifs de recherche (Allard-Poesi, DruckerGodard et Ehlinger, 2003).

\section{Résultats}

L'analyse des entrevues a permis d'identifier les parties prenantes de l'écosystème entrepreneurial impliquées dans chacune des phases du processus d'innovation, mais également leurs implications.

\subsection{Phase 1 : conception}

La première phase du processus simplifié d'innovation de Tiwari (2007) est la phase de conception. Elle comprend l'analyse des besoins, la génération et l'évaluation d'idées, ainsi que la planification du projet. Les entreprises étudiées ont réalisé ou réalisent actuellement cette phase de conception. L'analyse des entrevues a permis de révéler l'implication des parties prenantes dans cette première phase puisque les répondants ont mentionné les parties prenantes qui y ont été impliquées. Un seul répondant a mentionné l'implication d'aucune partie prenante dans cette phase. À l'opposé de ce dernier, un répondant a identifié l'implication de 9 parties prenantes dans son projet d'innovation. Bref, l'ensemble des répondants ont identifié l'implication de 11 parties prenantes dans cette phase de conception.

Une des parties prenantes qui a été grandement mentionnée par les répondants est la famille. Cette dernière permet de valider les idées d'innovation des entrepreneurs. Elle représente un partenaire d'affaires privilégié, car elle fournit du soutien moral et souvent financier. Pour certains répondants, la famille a fourni un logement permettant à ces entrepreneurs technologiques de concentrer leurs efforts sur leur projet. Pour un autre répondant, la famille l'a encouragé à suivre des formations et des séminaires.

L'analyse des résultats des entrevues a également révélé que les fournisseurs prenaient une place importante dans cette phase. Ces derniers foumissent non seulement des composants, mais également de l'aide technique ainsi que des infrastructures facilitant la conception d'un projet d'innovation technologique. Les associations professionnelles ainsi que les cabinets comptables donnent, quant à eux, accès à des réseaux de contacts permettant d'identifier des clients potentiels. Les répondants ont également mentionné que leurs clients leur permettent de réaliser des expériences techniques sur leur projet technologique.

De plus, les universités et les centres collégiaux de transfert de technologie ont un rôle important dans la phase de conception. En effet, ces derniers procurent aux entrepreneurs des services de conseillers spécialisés de manière à fournir de l'aide technique permettant de mieux évaluer le marché et de mieux orienter leur projet d'innovation technologique. Parmi les parties 
prenantes impliquées dans cette phase, les répondants ont également mentionné les avocats, qui gèrent légalement les ententes, ainsi que les agents de brevet, qui fournissent des informations et réalisent des études de brevetabilité.

Cette étude a par ailleurs révélé que certains organismes gowvernementaux fournissent de l'aide financière liée à l'innovation. De plus, les répondants ont mentionné que les gestionnaires de capital de risque fournissent non seulement du soutien financier, mais également du mentorat ainsi qu'un accès à des infrastructures, à des équipements et à un réseau de contacts. Finalement, les incubateurs représentent une partie prenante importante dans cette phase de conception. Ils fournissent l'accès à du mentorat et à des compétences en marketing afin d'évaluer le potentiel de marché. Ils facilitent donc le partage de connaissances et soutiennent même la recherche de financement.

L'analyse des implications des parties prenantes lors de la phase de conception d'un projet d'innovation technologique a ainsi révélé quatre grands rôles de ces parties prenantes au sein de l'écosystème entrepreneurial :

1. Structurer le projet (universités et centres collégiaux de transfert de technologie, agents de brevet, incubateurs et avocats);

2. Apporter une contribution technique (universités et centres collégiaux de transfert de technologie, gestionnaires de capital de risque, clients et fournisseurs);

3. Construire un réseau de contacts (associations professionnelles, incubateurs, gestionnaires de capital de risque, cabinets comptables et famille);

4. Fournir du financement et/ou aider à la recherche du financement (famille, organismes gouvernementaux, gestionnaires de capital de risque et incubateurs).

\subsection{Phase 2 : mise en œuvre}

La deuxième phase du processus simplifié d'innovation de Tiwari (2007) est la phase de mise en œuvre. Elle porte essentiellement sur le développement de l'innovation incluant le prototype, le pilote ainsi que les tests liés à l'innovation. Parmi les répondants, 10 des 11 entreprises étudiées ont réalisé ou réalisent actuellement cette phase de mise en œuvre. Une seule entreprise n'a pas atteint cette phase.

L'analyse de ces 10 répondants a révélé l'implication de 9 parties prenantes dans cette phase. Par ailleurs,
8 des parties prenantes qui ont joué un rôle dans la phase de conception sont également impliquées dans la phase de mise en œuvre : les clients, les fournisseurs, les universités et les centres collégiaux de transfert de technologie, les avocats, les organismes gouvernementaux, les gestionnaires de capital de risque, la famille ainsi que les associations professionnelles.

Les implications de ces parties prenantes dans la phase de mise en œuvre sont diverses. Les clients fournissent des idées de nouvelles applications, tandis que les fournisseurs tentent d'apporter du soutien dans l'amélioration du produit ou encore dans les demandes de subvention. Les universités et les centres collégiaux de transfert de technologie visent, quant à eux, à fournir des compétences spécialisées en mettant des conseillers à la disponibilité des entreprises. Tout comme dans la phase de conception, les avocats aident les entreprises à gérer légalement les ententes. Les organismes gowvernementaux et les gestionnaires de capital de risque peuvent fournir du financement lors de la phase de mise en œuvre. La famille représente toujours un partenaire d'affaires privilégié. Les associations professionnelles, quant à elles, permettent aux entreprises d'accéder à un réseau de contacts ainsi qu’à des clients.

Bref, seuls les cabinets comptables, les agents de brevet et les incubateurs n'ont pas été cités lors des entrevues pour cette phase de mise en œuvre. Par contre, une nouvelle partie prenante s'implique dans cette deuxième phase: les consultants. Ces derniers fournissent des connaissances et des compétences spécialisées de manière à bien analyser la mise en œuvre du projet technologique.

L'analyse des implications des parties prenantes lors de la phase de mise en œuvre d'un projet d'innovation technologique a donc révélé quatre grands rôles de ces parties prenantes au sein de l'écosystème entrepreneurial :

1. Accéder à diverses ressources tangibles et intangibles (gestionnaires de capital de risque, universités et centres collégiaux de transfert de technologie, onganismes gowvernementaux, fournisseurs et consultants);

2. Accéder à des services (organismes gowvernementaux, avocats et consultants);

3. Accéder à un réseau de contacts (associations professionnelles, clients, famille, fournisseurs et universités et centres collégiaux de transfert de technologie);

4. Fournir du financement (famille, organismes gowvernementaux et gestionnaires de capital de risque). 


\subsection{Phase 3 : marketing}

La dernière phase du processus simplifié d'innovation de Tiwari (2007) est la phase de marketing. Cette phase comprend la production de l'innovation technologique ainsi que sa mise en marché, tant pour les marchés nationaux qu'internationaux, grâce à des techniques de commercialisation utiles à la pénétration de ces marchés.

Étant souvent des chercheurs ou des ingénieurs, les entrepreneurs technologiques possèdent plus de compétences pour la conception et la mise en œuvre d'un produit technologique que de compétences liées à la commercialisation (Albert, 2000). Ils ont généralement une bonne maitrise des connaissances techniques, mais ils éprouvent des difficultés lors de la commercialisation de leur innovation. En effet, les compétences en marketing sont plus rares chez les entrepreneurs technologiques (Borges, Filion et Simard, 2005).

Bien qu'il n'y ait seulement que 6 des 11 entreprises étudiées qui ont réalisé ou réalisent actuellement cette phase de marketing, l'analyse des entrevues a quand même révélé l'implication de 10 parties prenantes dans cette phase : les clients, les universités et les centres collégiaux de transfert de technologie, les avocats, les onganismes gouvernementaux, les incubateurs, les gestionnaires de capital de risque, les associations professionnelles, les consultants ainsi que les distributeurs et les institutions financières.

Ces deux dernières parties prenantes n'ont pas été identifiées dans les deux premières phases du processus d'innovation. Toutefois, elles jouent des rôles significatifs dans la phase de marketing. Les distributeurs facilitent la promotion et la distribution de l'innovation technologique, car ils possèdent des compétences spécifiques facilitant la commercialisation. Les institutions financières, quant à elles, entrent également en jeu dans cette dernière phase en fournissant du financement au projet d'innovation. Étant des créanciers et non des investisseurs, les institutions financières visent à financer des projets prêts à la commercialisation, et non à financer les preuves de concepts, les études de marché et les différents tests et prototypes.

L'analyse des implications des parties prenantes lors de la phase de marketing d'un projet d'innovation technologique a révélé quatre grands rôles de ces parties prenantes au sein de l'écosystème entrepreneurial :
1. Accéder à diverses ressources servant à la commercialisation (clients, universités et centres collégiaux de transfert de technologie, incubateurs, gestionnaires de capital de risque, associations professionnelles et consultants);

2. Accéder à des services liés aux activités de marketing (avocats, organismes gouvernementaux et distributeurs);

3. Accéder à un réseau de contacts (organismes gowvernementaux et associations professionnelles);

4. Fournir du financement (gestionnaires de capital de risque et institutions financières).

\section{Discussion et conclusion}

Cette recherche a permis, dans un premier temps, de réaliser que l'implication et le rôle des parties prenantes évoluent à mesure que le projet d'innovation technologique progresse. En effet, l'écosystème entrepreneurial semble s'adapter en fonction du degré d'avancement du projet technologique. Les entrevues ont révélé que certaines parties prenantes ne contribuent qu'à la phase de conception, soit les agents de brevet et les cabinets comptables. Par ailleurs, d'autres ne sont impliquées qu'à la phase de marketing : les distributeurs et les institutions financières. Certaines parties prenantes identifiées sont présentes dans plus d'une phase du processus simplifié de l'innovation (Tiwari, 2007), bien que leur rôle puisse être appelé à évoluer au fil de ces phases.

Par ailleurs, le tableau 2 portant sur l'évolution de l'écosystème entrepreneurial lors de projets technologiques montre l'évolution du rôle des parties prenantes au fil des phases du processus d'innovation d'une entreprise technologique. D'après les répondants, les entrepreneurs technologiques s'attardent davantage à l'aspect technique qu'à l'aspect managérial lors des phases de conception et de mise en œuvre. Par contre, l'aspect managérial, notamment les activités liées à la commercialisation, prend une ampleur plus importante lors de la phase de marketing. De plus, certains rôles, notamment celui du financement, sont joués par des parties prenantes différentes selon les phases de processus d'innovation. Par exemple, les incubateurs et les organismes gouvernementaux seront impliqués dans la recherche de financement dès le début du processus d'innovation. Contrairement à ces derniers, les institutions financières ne sont sollicitées que lors de la commercialisation. 


\begin{tabular}{|c|c|c|c|}
\hline \multirow[t]{2}{*}{ Parties prenantes } & \multicolumn{3}{|c|}{ Rôles dans le processus d'innovation } \\
\hline & Phase 1 : conception & Phase $2:$ mise en œuvre & Phase 3 : marketing \\
\hline Agents de brevet & - Structure du projet & & \\
\hline $\begin{array}{l}\text { Associations } \\
\text { professionnelles }\end{array}$ & - Réseautage & - Réseautage & $\begin{array}{l}\text { - Accès à des ressources } \\
\text { - Réseautage }\end{array}$ \\
\hline Avocats & - Structure du projet & - Accès à des services & - Accès à des services \\
\hline $\begin{array}{l}\text { Cabinets } \\
\text { comptables }\end{array}$ & - Réseautage & & \\
\hline Clients & - Contribution technique & - Réseautage & - Accès à des ressources \\
\hline Consultants & & $\begin{array}{l}\text { - Accès à des ressources } \\
\text { - Accès à des services } \\
\end{array}$ & - Accès à des ressources \\
\hline Distributeurs & & & - Accès à des services \\
\hline Famille & $\begin{array}{l}\text { - Réseautage } \\
\text { - Recherche de } \\
\text { financement }\end{array}$ & $\begin{array}{l}\text { - Réseautage } \\
\text { - Financement }\end{array}$ & \\
\hline Fournisseurs & - Contribution technique & $\begin{array}{l}\text { - Accès à des ressources } \\
\text { - Réseautage }\end{array}$ & \\
\hline $\begin{array}{l}\text { Gestionnaires de } \\
\text { capital de risque }\end{array}$ & $\begin{array}{l}\text { - Contribution technique } \\
\text { - Réseautage } \\
\text { - Recherche de } \\
\text { financement }\end{array}$ & $\begin{array}{l}\text { - Accès à des ressources } \\
\text { - Financement }\end{array}$ & $\begin{array}{l}\text { - Accès à des ressources } \\
\text { - Financement }\end{array}$ \\
\hline Incubateurs & $\begin{array}{l}\text { - Structure du projet } \\
\text { - Réseautage } \\
\text { - Recherche de } \\
\text { financement }\end{array}$ & & - Accès à des ressources \\
\hline $\begin{array}{l}\text { Institutions } \\
\text { financières }\end{array}$ & & & - Financement \\
\hline $\begin{array}{l}\text { Organismes } \\
\text { gouvernementaux }\end{array}$ & $\begin{array}{l}\text { - Recherche de } \\
\text { financement }\end{array}$ & $\begin{array}{l}\text { - Accès à des ressources } \\
\text { - Accès à des services } \\
\text { - Financement }\end{array}$ & $\begin{array}{l}\text { - Accès à des services } \\
\text { - Réseautage }\end{array}$ \\
\hline $\begin{array}{l}\text { Universités et } \\
\text { centres collégiaux } \\
\text { de transfert de } \\
\text { technologie }\end{array}$ & $\begin{array}{l}\text { - Structure du projet } \\
\text { - Contribution technique }\end{array}$ & $\begin{array}{l}\text { - Accès à des ressources } \\
\text { - Réseautage }\end{array}$ & - Accès à des ressources \\
\hline
\end{tabular}

Tableau 2-Évolution de l'écosystème entrepreneurial lors de projets technologiques

Bien que cette recherche soit exploratoire et que les résultats ne peuvent pas servir à une généralisation, l'analyse des répondants des 11 entreprises québécoises a permis d'identifier et de comprendre davantage les différents rôles des parties prenantes de l'écosystème entrepreneurial dans le processus d'innovation technologique. Cet écosystème entrepreneurial semble également évoluer avec les phases du processus d'innovation.

D'un point de vue managérial, ces résultats de recherche peuvent servir de lignes directrices pour les futurs entrepreneurs afin de savoir de quelle façon les parties prenantes peuvent être impliquées dans leur processus d'innovation technologique en fonction du degré d'avancement de leur projet.
Par ailleurs, la recherche de financement semble avoir un rôle particulièrement important dans ce processus. Plusieurs parties prenantes sont impliquées dans ce rôle particulier, notamment la famille, les organismes gouvernementaux, les gestionnaires de capital de risque, les incubateurs et les institutions financières. Il serait intéressant, lors de futures recherches, d'approfondir leur rôle respectif ainsi que les différentes stratégies alternatives de financement dans la cadre de projets d'entrepreneuriat technologique.

De plus, d'autres parties prenantes pourraient avoir été impliquées dans le processus d'innovation des 11 entreprises étudiées puisque l'approche méthodologique utilisée visait à ne pas guider les réponses, mais plutôt à laisser les répondants identifier euxmêmes leurs parties prenantes. Par conséquent, cette 
recherche ne permet pas de dresser une liste exhaustive des parties prenantes impliquées, ni de préciser le pourcentage d'utilisation de chacune des parties

prenantes. Il serait donc pertinent, lors de futures recherches, d'estimer ce pourcentage en fonction des différentes phases du processus d'innovation.

\section{RÉFÉRENCES}

Albert, P. (2000). La création d'entreprises de haute technologie. Dans T. Verstraete (dir.), Histoire d'entreprendre : les réalités de l'entrepreneuriat (p. 149-160). Paris, France : Éditions Management et Société.

Allard-Poesi, F., Drucker-Godard, C. et Ehlinger S. (2003). Analyses de représentations et de discours. Dans R.-A. Thiétart (dir.), Méthodes de recherche en management (2e éd., p. 449-475). Paris, France : Dunod.

Bailetti, T. (2012). Technology entrepreneurship: Overview, definition, and distinctive aspects. Technology Innovation Management Review, 2(2), 5-12. Repéré à https://timreview.ca/sites/default/files/article_PDF/Bailetti_TIMReview_February2012.pdf

Borges, C., Filion, L. J. et Simard, G. (2005). Entreprendre au Québec, c'est capital! : résultats de recherches sur la création d'entreprises (Cahier de recherche). Montréal, QC : HEC Montréal, Chaire d'entrepreneuriat Rogers-J.-A.-Bombardier. Repéré à http://expertise.hec.ca/chaire_entrepreneuriat/wp-content/uploads/2005-20-rrcerapport2004-2005.pdf

Garud, R. et Karnøe, P. (2003). Bricolage versus breakthrough: Distributed and embedded agency in technology entrepreneurship. Research Policy, 32(2), 277-300. https://doi.org/10.1016/S0048-7333(02)00100-2

Gauthier, B. et Bourgeois, I. (2016). Recherche sociale : de la problématique à la collecte des données (6e éd.). Québec, QC : Presses de l’Université du Québec.

Jelinek, M. (1996). “Thinking technology” in mature industry firms: Understanding technology entrepreneurship. International Journal of Technology Management, 11(7-8), 799-813.

Jones-Evans, D. (1995). A typology of technology-based entrepreneurs: A model based on previous occupational background. International Journal of Entrepreneurial Behavior \& Research, 1(1), 26-47.

Liu, T.-H., Chu, Y.-Y., Hung, S.-C. et Wu, S.-Y. (2005). Technology entrepreneurial styles: A comparison of UMC and TSMC. International Journal of Technology Management, 29(1-2), 92-115. https://doi.org/10.1504/ijtm.2005.006006

Mason, C. et Brown, R. (2014). Entrepreneurial ecosystems and growth oriented entrepreneurship. (Rapport final). Paris, France : OECD. Repéré à www.oecd.org/cfe/leed/Entrepreneurial-ecosystems.pdf

Miles, M. B. et Huberman, M. A. (2005). Analyse des données qualitatives (2e éd.). Paris, France : De Boeck.

Muldoon, J., Bauman, A. et Lucy, C. (2018) Entrepreneurial ecosystem: Do you trust or distrust? Journal of Enterprising Communities, 12(2), 158-177. https://doi.org/10.1108/JEC-07-2017-0050

Nichols, S. P. et Armstrong, N. E. (2003). Engineering entrepreneurship: Does entrepreneurship have a role in engineering education? IEEE Antennas and Propagation Magarine, 45(1), 134-138. https://doi.org/10.1109/MAP.2003.1189659

Project Management Institute. (2008). PMBOK Guide: A guide to the project management book of knowledge (4e éd.). Newtown Square, PA: Project Management Institute.

Shane, S. et Venkataraman, S. (2003). Guest editors' introduction to the special issue on technology entrepreneurship. Research Policy, 32(2), 181-184. https://doi.org/10.1016/S0048-7333(02)00104-X

Thiétart, R.-A. (2014). Méthodes de recherche en management (4e éd.). Paris, France : Dunod.

Tiwari, R. (2007). The early phases of innovation: Opportunities and challenges in public-private partnership. Asia Pacific Tech Monitor, 24(1), 32-37. Repéré à https://ssrn.com/abstract=1583822

Venkataraman, S. et Sarasvathy, S. D. (2001). Strategy and entrepreneurship: Outlines of an untold story. Dans M. A. Hitt, E. Freema et J. S. Harrison (dir.), The Blackwell Handbook of Strategic Management (p. 650-668). Oxford, R.-U. : Wiley-Blackwell.

Wirtz, B. W. et Daiser, P. (2018). Business model innovation processes: A systematic literature review. Journal of Business Models, 6(1), 40-58. https://doi.org/10.5278/ojs.jbm.v6i1.2397 